\title{
Biophysical Reviews publications on DNA structure and function that complement this Special Issue on DNA supercoiling
}

\author{
Cris dos Remedios ${ }^{1}$
}

Received: 17 June 2016 / Accepted: 20 June 2016 / Published online: 15 July 2016

(C) International Union for Pure and Applied Biophysics (IUPAB) and Springer-Verlag Berlin Heidelberg 2016

Although this Special Issue provides an in-depth analysis of the importance of DNA supercoiling, I would like to refer readers to several other relevant reviews published in Biophysical Reviews on the wider topic of DNA structure and function.

In 2009 Srinivasan et al. reviewed the importance of the chemical structure of the nucleic acid bases to the polymeric properties of DNA. These authors focussed on the important contribution of $a b$ initio computations to our understanding of the importance of nucleotide sequence dependence in the accumulation of charge on the major- and minor-groove edges of the Watson-Crick base pairs. They showed how optimizing the base-pair planes of DNA with the same handedness influences the binding of proteins to DNA.

Three years later, this same laboratory (Olson et al. 2012) extended their computer simulations by highlighting the importance of communication between regulatory proteins attached to precisely constructed stretches of chromatin DNA. They developed new computational methods that provided insights into the roles played by individual proteins in reading and compacting the genome. As a consequence they were able to simulate the dynamic, three-dimensional structures of long, fluctuating, protein-decorated strands of DNA and take account of novel experimental measurements of enhancer-protein interactions. The states of chromatin captured in these simulations offered new insights into the ways DNA, histones

Cris dos Remedios

crisdos@anatomy.usyd.edu.au

1 Discipline of Anatomy \& Histology, Bosch Institute, Anderson Stuart Building, University of Sydney, Sydney 2006, Australia and regulatory proteins contribute to long-range communication along the genome.

The next year Biophysical Reviews reported (Qin and Zhou 2013) a freely-available suite of software, $\mathrm{PI}^{2} \mathrm{PE}$ (http://pipe. sc.fsu.edu) for predicting, inter alia, solvent accessibility of amino acids involved in protein folding and in protein-DNA complexes, as well as the binding rate constants of these complexes. The authors now have a new server that can predict these binding rate constants. These powerful web servers form a virtual pipeline between amino acid sequence data, tertiary structure, quaternary structure, and binding kinetics.

Last year Yuri Lyubchenko (Lyubchenko 2015) reviewed the contributions of single-molecule time-lapse atomic force microscopy (AFM) to our understanding of the accessibility of DNA within complex bodies, called nucleosomes (these form the fundamental repeating units of eukaryotic chromatin). It turns out that these structures, which were once thought to be static, are in fact highly dynamic when examined on a millisecond time scale. They exhibit sliding and unwrapping, and even complete dissociation. Thus, AFM can provide new insights into the mechanisms that control spontaneous chromatin dynamics.

Finally, in a hot-off-the-press review, Lois Pollack (Tokuda et al. 2016) reviews the way contrast variation in small angle X-ray scattering (SAXS) reveals new structural information on protein-DNA and ion-DNA interactions. Since DNA is essentially a long polyanion, its electrostatic interactions are mediated either by positively charged protein residues or by charge-compensating cations. SAXS is uniquely sensitive to both the conformation and local environment of its protein binding partners and associated ions that bind to DNA. Solution contrast variation is a technique that enables the measurement of DNA 
conformation within a protein-DNA complex by masking the contribution of the protein to the scattering profile. In this review, the authors show how SAXS reveals real-time unwrapping of DNA from a nucleosome. They also review how heavy-atom isomorphous replacement can be used to examine the spatial distribution of the cation "cloud" around a standard 25-base pair fragment of DNA by exploiting changes in the X-ray scattering strength of these cations.

With that background, I hope readers enjoy this Special Issue of Biophysical Reviews on "DNA supercoiling, protein interactions and genetic function" edited by Special Editor Laura Finzi and Executive Editor Wilma Olson.

Cris dos Remedios

Editor-in-Chief

\section{References}

Lyubchenko YL (2015) Nanoscale nucleosome dynamics assessed with time-lapse AFM. Biophys Rev 6(2):181-190

Olson WK, Clauvelin N, Colasanti AV, Singh G, Zheng G (2012) Insights into gene expression and packaging from computer simulations. Biophys Rev 4(3):171-178

Qin S, Zhou HX (2013) PI2PE: a suite of web servers for predictions ranging from protein structure to binding kinetics. Biophys Rev 5(1):41-46

Srinivasan AR, Sauers RR, Fenley MO, Boschitsch AH, Matsumoto A, Colasanti AV, Olson WK (2009) Properties of the nucleic acid bases in free and Watson-Crick hydrogen-bonded States: computational insights into the sequence-dependent features of double-helical DNA. Biophys Rev 1(1):13-20

Tokuda JM, Pabit SA, Pollack L (2016) Protein-DNA and ion-DNA interactions revealed through contrast variation SAXS. Biophys Rev 8(2):139-149 